OnLine Journal of Biological Sciences 11 (3): 84-89, 2011

ISSN 1608-4217

(C) 2011 P. Zareian et al., This open access article is distributed under a Creative Commons Attribution

(CC-BY) 3.0 license

\title{
Interleukin-10, Mean Arterial Blood Pressure and Insulin Resistance in Normal Pregnancy
}

\author{
${ }^{1,2}$ Abdolreza Sotoodeh Jahromi, ${ }^{1}$ Mohammad Reza Farjam, \\ ${ }^{2}$ Mohammad Hassan Davami, ${ }^{3}$ Alireza Makarem, \\ ${ }^{3}$ Mohammad-Yasin Karami, ${ }^{4}$ Parvin Zareian and ${ }^{5}$ Abdolhossein Madani \\ ${ }^{1}$ Department of Immunology, \\ ${ }^{2}$ Research Center of Zoonosis Diseases, \\ ${ }^{3}$ Department of Research Committee, \\ ${ }^{4}$ Department of Physiology, \\ Jahrom University of Medical Sciences, Jahrom, Iran \\ ${ }^{5}$ Department of Epidemiology, Center for Determinants in Health Promotion, \\ Hormozgan University of Medical Science, Bandarabbas, Iran
}

\begin{abstract}
Problem statement: The purpose of this study was to investigate whether serum of Interleukin-10 (IL-10) Concentration change during normal pregnancy and, if so, to relate these changes corresponding alterations in insulin resistance and blood pressure. Approach: This cross sectional study was carried out on 86 healthy pregnant women including 26, 23 and 37 individuals in the 1st, 2nd and 3rd trimesters, respectively and in 21 healthy non pregnant women. Serum IL-10 concentration was measured by Enzyme Linked Immunosorbent Assay (ELISA) method. Insulin resistance value was calculated using the homeostasis model assessment, HOMA-IR. Results: Serum IL-10 concentration was found to be significantly higher in patients in all gestational age as compared non pregnant women. Il-10 level was significantly increased with increase in gestational age. Pregnant women exhibited higher score of HOMA IR compared non pregnant women, but there were no difference in this score between pregnant subjects in different gestational age. There were not significant correlation between IL-10 level with IR and blood pressure. Conclusion: The results of the study show maternal IL-10 level increase with further increase in gestational age and there is no significant correlation between IL-10 level with Mean Arterial blood Pressure (MAP) and IR.
\end{abstract}

Key words: Enzyme Linked Immunosorbent Assay (ELISA), Mean Arterial blood Pressure (MAP), changes corresponding alterations, between pregnant subjects, Insulin Resistance (IR)

\section{INTRODUCTION}

Normal pregnancy is associated to change in proinflammatory and anti-inflammatory cytokines (Davila et al., 2011; Russell et al., 1997; Holmes et al., 2003). Cytokine balance is important for normal processes of pregnancy and onset of labor (Arntzen et al., 1997).

Interleukin-10 (IL-10) is an anti-inflammatory and immunomodulatory $\mathrm{Th} 2$ cytokines, that produce by a range of maternal and fetal cells (Vigano et al., 2001; Hennessy et al., 2003). IL-10 has a critical role in maintaining the balance of anti-inflammatory and proinflammatory milieu at the maternal-fetal interface (Kalkunte et al., 2011). Metabolic Syndrome (MS) is a collection of risk factors including insulin resistance, central obesity, hypertension and dyslipidemia and itself is a risk factor for coronary artery disease (Mahajan et al., 2010). Pregnancy is associated to glucose metabolism disorders, Insulin Resistance (IR) (Hadden and McLaughlin, 2009; Johnson, 2008) and oxidative stress (Idonije et al., 2011). The Insulin Resistance syndrome is comprised of a cluster of metabolic abnormalities that confer increased risk of diabetes, hypertension and cardiovascular disease (Grinspoon, 2006). Insulin Resistance (IR) may facilitate supply of appropriate nutrients particularly of glucose to fetus for fetal growth and metabolism. The mechanism responsible for insulin resistance has not been clearly stated. Recent researches have been shown IL-10 increase insulin sensitivity (Hong et al., 2009; Ye et al., 2006; Holmes et al., 2003; Straczkowski et al., 2005). Insulin sensitivity changes from an enhance state during early pregnancy to an insulin resistant state in late pregnancy (Kirwan et al., 2002). 
Insulin resistance may facilitate supply of appropriate nutrients particularly of glucose to fetus for fetal growth and metabolism. The mechanism responsible for insulin resistance has not been clearly stated. Insulin resistance can be affected by Nitric Oxide (NO) (McGrowder and Brown, 2007). Recent researches have been shown adipokinins include leptin (Soheilykhah et al., 2011; Mohiti et al., 2009; Assal et al., 2007; EL-Ghaffar and EL-Said, 2006), resistin (Caja et al., 2005) and cytokines include TNf- $\alpha$ (Kirwan et al., 2002), IL-6 and IL-1ß (Nov et al., 2010) play an important role in insulin resistance. Insulin sensitivity changes from an enhanced state during early pregnancy to an insulin resistant state in late pregnancy. Insulin sensitivity changes from an enhanced state during early pregnancy to an insulin resistant state in late pregnancy (Kirwan et al., 2002).

Therefore, it is inspected, subsequent to change in insulin resistance during normal pregnancy, IL-10 level change too. In a recently published study, it has been reported that in normal pregnant women both maternal IL-6 (Jahromi et al., 2011a) and TNF- $\alpha$ cytokines are increased (Zareian et al., 2010; Elsamanoudy and Abbas, 2010) and IL-1 $\beta$ is decreased (Jahromi et al., 2011b), But it was not find any correlation between IR with IL-6 and TNF- $\alpha$. (Jahromi et al., 2011a) and between IR with IL-1 $\beta$ (Jahromi et al., 2011b). Resistin is an insulin-sensitizing adipokine that can be implicated in endogenous glucose regulation (Mohiti et al., 2009).

However, at the time of this study data concerning the pattern of IL-10 secretion in normal pregnancies are still limited and partially contradictory (Russell et al., 1997) and its correlation with insulin resistance during normal pregnancy have not been studied. Therefore, the aim of this study was examine either serum IL-10 concentration change during normal pregnancy and if so, to relate those changes corresponding alterations in insulin resistance, mean arterial pressure and body mass index.

\section{MATERIALS AND METHODS}

This study was conducted at the department of Obstetrics and Gynecology of Honary Clinic, Jahrom, Iran. Subjects were 86 pregnant women with different gestational ages (first trimester: $26 \mathrm{sec}$ trimester: 23, third trimester: 37 and 21 non pregnant women similar in age and Body Mass Index (BMI). All subjects met the following criteria: no history of pre- gestational diabetes; no history of liver, respiratory, thyroid or other illness and any current infectious condition. They were not on any drug therapy.
Body Mass Index (BMI, $\mathrm{Kg} \mathrm{m}^{-2}$ ) was calculated according to the maternal height and pre-pregnancy weight. Serum samples were analysed for concentrations of IL-10, insulin and glucose. Blood sugar was measured by Glucose Oxidase/Peroxidase (GOD-POD) method. Serum insulin was determined by ELISA (Diaplus; based on the direct sandwich technique in which two monoclonal antibodies are directed against separate antigenic determinants on the insulin molecule. During incubation insulin in the sample reacts with enzyme (HRP) conjugated antiinsulin antibody and anti-insulin antibody bound to micro-titration well. A sample washing step removed unbound enzyme labeled antibody. In the insulin ELISA, the bound HRP complex is detected by reaction with TMB substrate. The reaction is stopped by adding acid to give a colorimetric endpoint that is read using ELISA reader). Serum IL-10was measured by ELISA (Bendermed, Austria: Cat. No. BMS224INSTCE). An anti-human IL-10 coating antibody is adsorbed onto microwells. Human IL-10 present in the sample or standard binds to antibodies adsorbed to the microwells; a biotin-conjugated monoclonal anti-human IL-10 antibody binds to human IL-10 captured by the first antibody. Streptavidin-HRP binds to the biotin conjugated anti-human IL-10. Following incubation unbound biotin conjugated anti human IL-10 and Streptavidin-HRP is removed during a wash step and substrate solution reactive with HRP is added to the wells. A colored product is formed in proportion to the amount of soluble human IL-10 present in the sample. The reaction is terminated by addition of acid and absorbance is measured at $450 \mathrm{~nm}$. A standard curve is prepared from seven human IL-10 standard dilutions and human IL-10 sample concentration determined.

Mean Arterial Pressure (MAP) was calculated by formula as (Systolic pressure $\mathrm{mmHg}$ ) + (Diastolic pressure $^{2} \mathrm{mmHg} / 3$.

Insulin resistance value were calculated using the homeostasis model assessment, HOMA- IR ,as (fasting insulin IU/L $) \times\left(\right.$ fasting glucose $\left.\mathrm{mmol} \mathrm{L}^{-1}\right) / 22.5$ as previously reported by (Matthews et al., 1985).

Statistical analysis: All results are displayed as Mean \pm SD (standard deviation of mean) and Min-Max. Resistin, Insulin Resistance (IR), BMI and body weight data were analyzed with One Way Analysis of Variance (ANOVA). Serum IL-1 $\beta$ and insulin concentration data were analyzed with non-parametric kruskal-wallis test (Vargha, 1998) followed by Mann Whitney U-test 
(Rosner and Grove, 1999). Correlations were calculated using liner correlation (pearson). Statistical analysis was performed using SPSS 11 for windows. $p<0.05$ was considered statistically significant for all analysis.

\section{RESULTS}

A total of 86 pregnant women and 21 non pregnant subjects participated in the study. Clinical and laboratory characteristics of pregnant women and controls are summarized in Table 1. BMI was found to be significantly increased in the $3 \mathrm{rd}$ trimester as compared with controls and women with 1st trimester of pregnancy Fig. 1a, Table 2. Pregnant women in the second and third trimester of pregnancy had significantly higher systolic pressure than non pregnant women Fig. 1b. IL-10 level was also significantly higher in patients in all gestational age as compared non pregnant women Fig. 1c. However, during pregnancy IL-10 level were significantly increased with increase in gestational age Table 2 Pregnant women exhibited higher score of HOMA IR compared non pregnant women, but there were no difference in this score between pregnant subjects in different gestational age Table 1 and 2 . There were significant correlation between gestational age with BMI $(r=0.28, p=0.01)$, diastolic pressure $(r=0.28, p=0.01)$ and IL-10 level $(r$ $=0.56, \mathrm{p}<0.01)$. There was not significant correlation between gestational age and IR. IL-10 level also correlates with BM $(r=0.299, p=0.004)$.

There were not significant correlation between IL10 level with IR, Systolic pressure, diastolic pressure, MAP and fasting insulin level.

Table 1: Clinical and laboratory characteristic of patients and control

\begin{tabular}{|c|c|c|}
\hline & Pregnant women & Control \\
\hline Number of case & 86 & 21.0 \\
\hline Age (year) & $26.4 \pm 4.1$ & $27.2 \pm 5.6$ \\
\hline Gestational age (week) & $23.9 \pm 9.8$ & \\
\hline HT (m) & $1.6 \pm 0.06$ & $1.58 \pm 0.07$ \\
\hline WT (Kg) & $64.99 \pm 11.5 b$ & $58.6 \pm 6.4$ \\
\hline $\mathrm{BMI}\left(\mathrm{Kg} \mathrm{m}^{-2}\right)$ & $25.4 \pm 3.7 \mathrm{a}$ & $23.4 \pm 3$ \\
\hline $\mathrm{SBP}(\mathrm{mmHg})$ & $117 \pm 7.8 \mathrm{~b}$ & $110.7 \pm 5.9$ \\
\hline $\mathrm{DBP}(\mathrm{mmHg})$ & $72.8 \pm 6.9$ & $70.9 \pm 11.4$ \\
\hline MAP $(\mathrm{mmHg})$ & $86.5 \pm 6.70$ & $85.1 \pm 8.4$ \\
\hline $\mathrm{BGL}\left(\mathrm{mg} 100^{-1}\right)$ & $81.5 \pm 8.7$ & $80.2 \pm 8.7$ \\
\hline Insulin $\left(\mu \mathrm{LU} \mathrm{mL} \mathrm{m}^{-1}\right)$ & $10.9 \pm 6.00$ & $8.7 \pm 1.9$ \\
\hline IL-10 (pg mL $\left.{ }^{-1}\right)$ & $0.851 \pm 0.702 \mathrm{c}$ & $0.119 \pm 0.087$ \\
\hline IR & $2.1 \pm 0.7 \mathrm{a}$ & $1.7 \pm 0.4$ \\
\hline
\end{tabular}

BMI: Body Max Index, HT: Height of Women, WT: Weight of body SBP: Systolic blood pressure DBP: Diastolic blood pressure MAP: mean arterial pressure BGL: Blood glucose level IL-10: Interleukin10 IR: Insulin resistance ${ }^{\mathrm{a}} \mathrm{p}<0.05$ (control) ${ }^{\mathrm{b}} \mathrm{p}<0.01 \quad\left(\right.$ control) ${ }^{\mathrm{c}}$ $\mathrm{p}<0.0001$ (control)

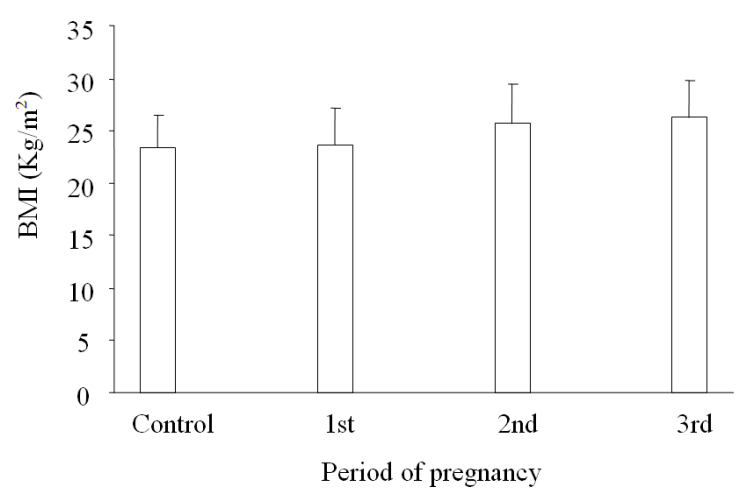

(a)

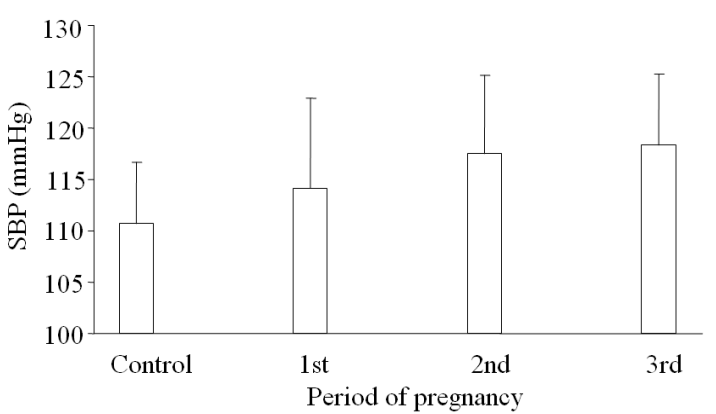

(b)

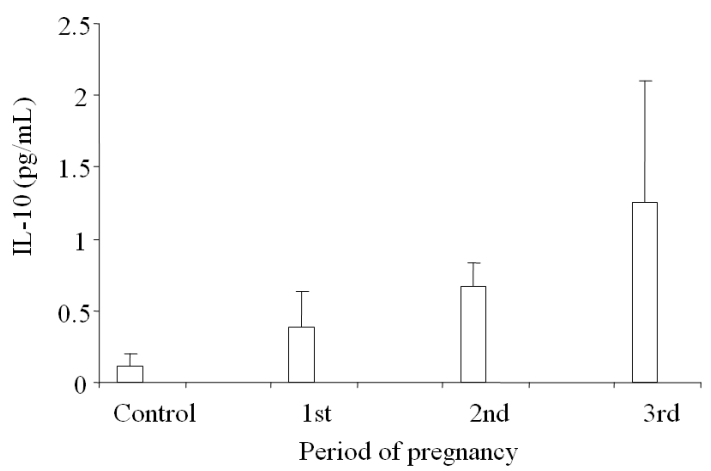

(c)

Fig. 1:(A) BMI in patients in different trimesters of pregnancy and controls. BMI were significantly higher in 3rd trimesters compared with the control (b: p<0.01). (B) Systolic blood pressure in different trimesters of pregnancy and controls. Systolic blood pressure were significantly higher in the 2nd and 3rd trimesters as compared with control group (a: $\mathrm{p}<0.05$, b: $\mathrm{p}<0.01)$. (C) Serum IL-10 level in different trimesters of pregnancy and control. Serum IL-10 level significantly higher in the 1st, 2nd and 3rd trimester as compared control (a: $p<0.05$, b: $p<0.01$, c: $\mathrm{p}<0.001)$ 
OnLine J. Biol. Sci., 11 (3): 84-89, 2011

Table 2: Clinical and laboratory characteristics of pregnant women with different gestational age

\begin{tabular}{|c|c|c|c|}
\hline & $\begin{array}{l}\text { 1st trimester } \\
\text { Mean } \pm \mathrm{SD}\end{array}$ & $\begin{array}{l}\text { 2nd trimester } \\
\text { Mean } \pm \text { SD }\end{array}$ & $\begin{array}{l}\text { 3rd trimester } \\
\text { Mean } \pm \text { SD }\end{array}$ \\
\hline Number of cases & 26 & 23 & 37.00000 \\
\hline Age (year) & $25.5 \pm 4.1000$ & $24.9 \pm 2.3000$ & $27.6 \pm 4.7000$ \\
\hline GA (week) & $11.2 \pm 1.6000$ & $22.2 \pm 2.7000$ & $32.9 \pm 4.3000$ \\
\hline WT (Kg) & $59.2 \pm 10.200$ & $65.7 \pm 12.600$ & $68.2 \pm 10.6 \mathrm{~b} 0$ \\
\hline HT (m) & $1.58 \pm 0.0600$ & $1.58 \pm 0.0600$ & $1.6 \pm 0.0700$ \\
\hline $\mathrm{SBP}(\mathrm{mmHg})$ & $114.2 \pm 8.700$ & $117.5 \pm 7.700$ & $118.3 \pm 7 \mathrm{a} 000$ \\
\hline $\mathrm{DBP}(\mathrm{mmHg})$ & $70.5 \pm 6.6000$ & $71.7 \pm 7.1000$ & $74.7 \pm 6.5000$ \\
\hline $\mathrm{MAP}(\mathrm{mmHg})$ & $85.1 \pm 6.9000$ & $86.7 \pm 6.9000$ & $89.4 \pm 6.2000$ \\
\hline $\mathrm{BMI}\left(\mathrm{Kg} \mathrm{m}^{-2}\right)$ & $23.6 \pm 3.6000$ & $25.7 \pm 3.7000$ & $26.2 \pm 3.6 \mathrm{~b} 00$ \\
\hline BGL (mg 100-1) & $78.6 \pm 6.7000$ & $79.4 \pm 6.7000$ & $84.1 \pm 21.200$ \\
\hline Insulin $\left(\mu \mathrm{L} \mathrm{mL}^{-1}\right)$ & $10.5 \pm 3.4000$ & $10.6 \pm 3.8000$ & $10.05 \pm 3.900$ \\
\hline $\mathrm{IL}-10\left(\mathrm{pg} \mathrm{mL}^{-1}\right)$ & $0.383 \pm 0.247$ & $0.672 \pm 0.156$ & $1.257 \pm 0.847 \mathrm{~b}$ \\
\hline IR & $2 \pm 0.600000$ & $2.1 \pm 0.60000$ & $2.1 \pm 0.80000$ \\
\hline
\end{tabular}

BMI: Body mass index GA: Gestational age WT: Wight of body HT: Height SBP: Systolic blood pressure DBP: Diastolic blood pressure MAP: Mean Arterial Pressure BGL: Blood Glucose Level IR: Insulin Resistance IL-10: Interleukin-10 ${ }^{a} p<0.05, \quad{ }^{b} p<0.01, \quad{ }^{c} p<0.001$ (Significantly different from pregnant women in $1^{\text {st }}$ trimester)

\section{DISCUSSION}

Glucose metabolism disorder is a common complication during pregnancy and its pathology is associated with IR and deficiency of insulin secretion2.In this study, insulin resistance significantly was higher in total group of healthy pregnant than in non pregnant women. In spite of previous report we did not found correlation between gestational age and insulin resistance. Previous researches have shown insulin resistance was significantly increased in late pregnancy compared with either control or early pregnancy (Kirwan et al., 2002; Melczer et al., 2002). This difference may be related to differences in dietary composition, life style between western and eastern societies (Clapp, 2006; Sivabalan et al., 2008), variability between insulin assays in different experimental researches, differences in the population studied and sampling time during pregnancy (Manley et al., 2008).

Cytokine proteins are soluble proteins that involved in the inflammation and many other processes. These proteins classifies based on their T-cell linage as Th1 or Th2. There are reports demonstrated that in normal pregnant women circulating $\mathrm{Th} 2$ cell-derived cytokines increased and Th1 cell derived cytokines decreased (Saito et al., 1999).

The results of present study are in consistent with a previous similar study that found an increase in IL-10 level in pregnant compared nonpregnant subjects (Holmes et al., 2003), but our study showed a significant correlation between serum IL-10 concentration and gestational age. Maternal serum IL-10 increased with further increase in pregnancy period. It has been indicated that Il-10 has a role in regulating placental development and programming (Roberts et al., 2003). Furthermore, the derived cytokine as IL-10 may be associated to normal human pregnancy and labor (Opsjłn et al., 1993). Test of correlation showed significant positive correlation between IL-10 level and BMI. Therefore, it seems, IL10 produces not only by intrauterine tissue but also extra uterine tissues (Schaffler et al., 2006).

In a previous study, have been shown that administration of the IL-10 can normalize blood pressure in pregnancy-induced hypertensive rats (Tinsley et al., 2010). In other study antihuman IL-10 monoclonal antibody administration to baboons in early pregnancy, caused a significant increase in mean arterial pressure (Orangea et al., 2005).

Based on these observations it will be speculated that with further increase in IL-10 production during pregnancy period blood pressure decreases progressively, but the reports of the current study do not support of this speculation. In the study, in spite of significant increase in IL-10 level in pregnant compared nonpregnant subjects, MAP did not change significantly and there was a marginally significant correlation between IL-10 level and MAP in pregnant women $(r=0.22, p=0.053)$. Therefore, it seems endogenous production of IL-10 during normal pregnancy has a week effect on MAP

In our study, we could not find correlation between maternal IL-10 and insulin resistance.

\section{CONCLUSION}

In conclusion, the results of the study show maternal IL-10 level increases with further increase in gestational age. There is no significant correlation between IL-10 level with MAP and insulin resistance.

\section{REFERENCES}

Arntzen, K.J., E. Lien and R. Austgulen, 1997. Maternal serum levels of interleukin-6 and clinical characteristics of normal delivery at term. Acta Obstet. Gynecol. Scand., 76: 55-60. PMID: 9033245

Assal, H.S., M. Fath-Allah and A. Elsherbiny, 2007. Serum leptin and adiponectin in obese diabetic and non-diabetic. J. Med. Sci., 7: 865-869.

Caja, S., I. Martinez, M. Abelenda and M. Puerta, 2005. Resistin expression and plasma concentration peak at different times during pregnancy in rats. J. Endocrinol., 185: 551-559. PMID: 15930181

Clapp, J.F., 2006. Effects of diet and exercise on insulin resistance during pregnancy. Metab Syndr. Relat. Disord., 4: 84-90. PMID: 18370754 
Davila, R.D., C.G. Julian, M.J. Wilson, V.A. Browne and C. Rodriguez et al., 2011. Do cytokines contribute to the Andean-associated protection from reduced fetal growth at high altitude. Reprod. Sci., 18: 79-87. PMID: 20841404

EL-Ghaffar, N.A. and N.H. Said, 2006. Hypoadiponectinaemia in Egyptian patients with type ii diabetes mellitus with vascular complications. J. Med. Sci., 6: 626-630.

Elsamanoudy, A.Z. and A.M. Abbas, 2010. Endothelial dysfunction and insulin resistance as pathophysiologic mechanisms in a rat model of preeclampsia. Am. J. Biochem. Biotechnol., 6: 172-180. DOI: 10.3844/ajbbsp.2010.172.180

Grinspoon, S., 2006. Abnormal glucose regulation and treatment strategies for insulin resistance hivinfected patients. Am. J. Infect. Dis., 2: 184-186. DOI: 10.3844/ajidsp.2006.184.186

Hadden, D.R. and C. McLaughlin, 2009. Normal and abnormal maternal metabolism during pregnancy. Seminars Fetal Neonatal Med., 14: 66-71. PMID: 18986856

Hennessy, A., D.M. Painter, S. Orange and J.S. Horvath, 2003. Placental tissue interleukin-10 receptor distribution in pre-eclampsia. Am. J. Reprod. Immunol., 49: 377-381. PMID: 12951965

Holmes, V.A., W.J. Gilmore, W.S.P McFaul and H.D. Alexander, 2003. Plasma levels of the immunomodulatory cytokine interleukin-10 during normal human pregnancy: A longitudinal study. Cytokine, 21: 265-269. DOI: 10.1016/S10434666(03)00097-8

Hong, E.G., H.J. Ko, Y.R. Cho, H.J. Kim and Z. Ma et al., 2009. Interleukin-10 prevents diet-induced insulin resistance by attenuating macrophage and cytokine response in skeletal muscle. Diabetes, 58: 2525-2535. PMID: 19690064

Idonije, O.B., O. Festus, O. Okhiai and U. Akpamu, 2011. A comparative study of the status of oxidative stress in pregnant Nigerian women. Res. J. Obstetrics Gyn., 4: 28-36.

Jahromi, A.S., P. Zareian and A. Madani, 2011a. Association of insulin resistance with serum interleukin- 6 and TNF- $\alpha$ levels during normal pregnancy. Biomarker Insights, 6: 1-6.

Jahromi, A.S., P. Zareian and A. Madani, 2011b. Insulin resistance and interleukin-1 $\beta$ during normal pregnancy. Asian J. Biochem., 6: 366-372.

Johnson, C.A., 2008. Glucose homeostasis during canine pregnancy: Insulin resistance, ketosis, and hypoglycemia. Theriogenology, 70: 1418-1423. PMID: 18823654
Kalkunte, S., T. Nevers, W.E. Norris and S. Sharma, 2011. Vascular IL-10: A protective role in preeclampsia. J. Reprod. Immunol., 88: 165-169. PMID: 21334073

Kirwan, J.P., M.S. Hauguel-De, J. Lepercq, C. Challier and J. Huston-Presley et al., 2002. TNF-alpha is a predictor of insulin resistance in human pregnancy. Diabetes, 51: 2207-2213. PMID: 12086951

Mahajan, R., K. Gupta and V. Kapoor, 2010. A systematic account of pathogenesis, diagnosis and pharmacotherapy of metabolic syndrome: Things we need to know. Int. J. Pharmacol., 6: 338-345.

Manley, S.E., S.D. Luzio, I.M. Stratton, T.M. Wallace and P.M. Clark, 2008. Preanalytical, analytical, and computational factors affect homeostasis model assessment estimates. Diabetes Care, 31: 18771883. PMID: 18535197

Matthews, D.R., J.P. Hoskers, A.S. Rudenski, B.A. Naylor and D.F. Trencher et al., 1985. Homeostasis model assessment: insulin resistance and beta-cell function from fasting plasma glucose and insulin concentrations in man. Diabetologia, 28: 412-419. PMID: 3899825

McGrowder, D. and P.D. Brown, 2007. Effects of nitric oxide on glucose transport: In vivo and in vitro studies. Asian J. Biochem., 2: 1-18.

Melczer, Z., F. Banhidy, S. Csomor Jr, M. Kovacs and P. Siklos et al., 2002. Role of tumour necrosis factor- $\alpha$ in insulin resistance during normal pregnancy. Eur. J. Obstet. Gynecol . Reprod. Biol., 105: 7-10. DOI: 10.1016/S03012115(02)00108-2

Mohiti, J., F. Talebi and M. Afkhami-Ardekani, 2009. Circulation free leptin in diabetic patients and its correlation to insulin level. Pak. J. Biol. Sci., 12: 397-400. PMID: 19579977

Nov, O., A. Kohl, E.C. Lewis, N. Bashan and I. Dvir et al., 2010. Interleukin-1beta may mediate insulin resistance in liver-derived cells in response to adipocyte inflammation. Endocrinology, 151: 4247-4256. PMID: 20660063

Opsjłn, S..L, N.C. Wathen, S. Tingulstad, G. Wiedswang and A. Sundan et al., 1993 Tumor necrosis factor, interleukin-1, and interleukin- 6 in normal human pregnancy. Am. J. Obstet. Gynecol., 169: 397-404. PMID: 8362955

Orangea, S., J.E. Raskob, J.F. Thompsonc, J. Vaughand and E. Olived et al., 2005. Interleukin-10 regulates arterial pressure in early primate pregnancy. Cytokine, 29: 176-185. PMID: 15652450

Roberts, C.T., C.A. White, N.G. Wiemer, A. Ramsay and S.A. Robertson, 2003. Altered placental development in interleukin-10 null mutant mice. Placenta, 24: 94-99. PMID: 12842419 
Rosner, B. and D. Grove, 1999. Use of the MannWhitney U-test for clustered data. Stat. Med., 18: 1387-400. PMID: 10399203

Russell, A.S., C. Johnston, C. Chew and W.P. Maksymowych, 1997. Evidence for reduced Th1 function in normal pregnancy: A hypothesis for the remission of rheumatoid arthritis. J. Rheumatol., 24: 1045-1050. PMID: 9195507

Saito, S., M. Sakai, Y. Sasaki, K. Tanebe and H. Tsuda et al., 1999. Quantitative analysis of peripheral blood Th0, Th1, Th2 and the Th1:Th2 cell ratio during normal human pregnancy and preeclampsia. Clin Exp. Immunol., 117: 550-555. PMID: 10469061

Schaffler, A., A. Furst, C. Buchler, G. Paul and G. Rogler et al., 2006. Secretion of RANTES (CCL5) and interleukin-10 from mesenteric adipose tissue and from creeping fat in Crohn's disease: regulation by steroid treatment. J. Gastroenterol. Hepatol., 21: 1412-1418. PMID: 16911685

Sivabalan S. and V.P. Menon, 2008. Effect of feeding high-fat with or without sucrose on the development of diabetes in wistar Rats. Asian J. Biochem., 3: 271-279.

Soheilykhah, S., M. Mojibian, S. Rahimi-Saghand and M. Rashidi et al., 2011. Maternal serum leptin concentration in gestational diabetes. Taiwan J. Obstet. Gynecol., 50: 149-153. PMID: 21791299
Straczkowski, M., I. Kowalska, A. Nikolajuk, A. Krukowska and M. Gorska, 2005. Plasma interleukin-10 concentration is positively related to insulin sensitivity in young healthy individuals. Diabetes Care, 28: 2036-2037. PMID: 16043753

Tinsley, J.H., S. South, V.L. Chiasson and B.M. Mitchell, 2010. Interleukin-10 reduces inflammation, endothelial dysfunction, and blood pressure in hypertensive pregnant rats. Am. J. Physiol. Regu. Integr. Comp. Physiol., 298: 713719. PMID: 20053959

Vargha, A., 1998. The kruskal-wallis test and stochastic homogeneity. J. Educ. Behav. Stat., 23: 170-192. DOI: 10.3102/10769986023002170

Vigano, P., B. Gaffuri, E. Somigliana, M Infantino and M. Vignali et al., 2001. Interleukin-10 is produced by human uterine natural killer cells but does not affect their production of interferon- $\gamma$. Mol. Hum. Reprod., 7: 971-977. DOI: 10.1093/molehr/7.10.971

Ye, J.H., Z.Z. Li, Y. Li, F. Li and L. Yan et al., 2006. Relationship between serum interleukin-10 and insulin resistance in metabolic syndrome. Nan Fang Yi Ke Da Xue Xue Bao, 26: 428-430. PMID: 16624744

Zareian, P., A.S. Jahromi, F.H. Rad and S. Zarei, 2010. Do resistin and tumor necrosis factor- $\alpha$ relate to changes in insulin resistance in normal pregnancy? Am. J. Biochem. Biotechnol., 6: 19-24. DOI: 10.3844/ajbbsp.2010.19.24 